\title{
Effects of Inductance on the Propagation Delay and Repeater Insertion in VLSI Circuits
}

\author{
Yehea I. Ismail and Eby G. Friedman \\ Department of Electrical and Computer Engineering \\ University of Rochester \\ Rochester, New York 14627
}

\begin{abstract}
A closed form expression for the propagation delay of a CMOS gate driving a distributed $R L C$ line is introduced that is within $5 \%$ of dynamic circuit simulations for a wide range of $R L C$ loads. It is shown that the traditional quadratic dependence of the propagation delay on the length of an $R C$ line approaches a linear dependence as inductance effects increase. The closed form delay model is applied to the problem of repeater insertion in $R L C$ interconnect. Closed form solutions are presented for inserting repeaters into $R L C$ lines that are highly accurate with respect to numerical solutions. An $R C$ model as compared to an $R L C$ model creates errors of up to $30 \%$ in the total propagation delay of a repeater system. Considering inductance in repeater insertion is also shown to significantly save repeater area and power consumption. The error between the $R C$ and $R L C$ models increases as the gate parasitic impedances decrease which is consistent with technology scaling trends. Thus, the importance of inductance in high performance VLSI design methodologies will increase as technologies scale.
\end{abstract}

\section{Introduction}

It has become well accepted that interconnect delay dominates gate delay in current deep submicrometer VLSI circuits [1]-[5]. Currently, inductance is becoming more important with faster onchip rise times and longer wire lengths. Wide wires are frequently encountered in clock distribution networks and in upper metal layers. These wires are low resistance wires that can exhibit significant inductive effects. Furthermore, increasing performance requirements are pushing the introduction of new materials for low resistance interconnect [6]. With these trends it is becoming more important to include inductance when modeling on-chip interconnect. Criteria to determine which nets should consider on-chip inductance have been described in [7] and [8].

The focus of this paper is to provide an accurate estimate of the propagation delay of a CMOS gate driving a distributed $R L C$ line as well as to develop design expressions for optimum repeater insertion to minimize the delay of a signal propagating along a distributed $R L C$ line. The paper is organized as follows. In section II, a simple yet accurate propagation delay formula describing a CMOS gate driving a distributed $R L C$ load is presented. In section III, the propagation delay formula is used to develop design expressions for optimum repeater insertion to minimize the propagation delay of a

This research was supported in part by the National Science Foundation under Grant No. MIP-9610108, a grant from the New York State Science and Technology Foundation to the Center for Advanced Technology - Electronic Imaging Systems, and by grants from the Xerox Corporation, IBM Corporation, and Intel Corporation.

Permission to make digital/hardcopy of all or part of this work for personal or classroom use is granted without fee provided that copies are not made or distributed for profit or commercial advantage, the copyright notice, the title of the publication and its date appear, and notice is given that copying is by permission of ACM, Inc. To copy otherwise, to republish, to post on servers or to redistribute to lists, requires prior specific permission and/or a fee.

DAC 99, New Orleans, Louisiana

(c) 1999 ACM 1-58113-109-7/99/06..\$5.00 distributed $R L C$ line. Some conclusions are offered in section IV. A proof of the expressions for optimum repeater insertion in an $R L C$ line is provided in the appendix.

\section{Propagation Delay of a CMOS Gate Driving an $R L C$ Load}

An arbitrary CMOS gate driving an $R L C$ transmission line representation of an interconnect line is shown in Fig. 1. $R_{t}, L_{t}$, and $C_{t}$ are the total resistance, inductance, and capacitance of the line, respectively. The parasitic impedances $R_{t}, L_{t}$, and $C_{t}$ are given by $R_{t}$ $=R l, L_{t}=L l$, and $C_{t}=C l$, respectively, where $R, L$, and $C$ are the resistance, inductance, and capacitance per unit length of the interconnect and $l$ is the length of the line. $R_{t r}$ is the equivalent output resistance of the gate driving the interconnect. $C_{L}$ is the input capacitance of the following gate at the end of the interconnect section. A minimum size buffer has an output resistance $R_{0}$ and an input capacitance $C_{0}$. The input voltage $V_{\text {in }}$ is a fast rising signal that can be approximated by a step signal. $V_{\text {out }}$ is the far output voltage at the end of the interconnect section.

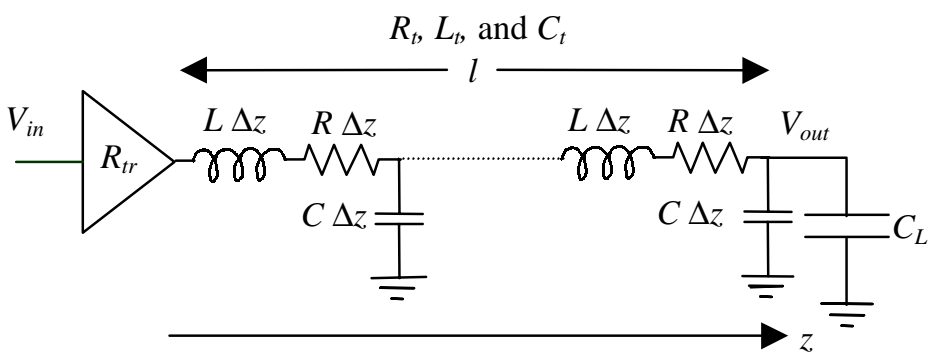

Fig. 1. A CMOS gate driving an $R L C$ transmission line.

From the basic principles of a transmission line [12], the voltage transfer function $V_{\text {out }}(S) / V_{\text {in }}(S)$ at the end of a lossy transmission line with a source resistance $z_{s}$ and a load impedance $z_{L}$ is given by

$$
\frac{V_{\text {out }}(S)}{V_{\text {in }}(S)}=\frac{2}{\left(\frac{z_{s}}{z_{0}}+1\right)\left(\frac{z_{0}}{z_{L}}+1\right) e^{\gamma}+\left(\frac{z_{s}}{z_{0}}-1\right)\left(\frac{z_{0}}{z_{L}}-1\right) e^{-\gamma}},
$$

where $\gamma$ is the propagation constant and $z_{0}$ is the characteristic impedance which are given by

$$
z_{0}=\sqrt{\frac{L_{t}}{C_{t}}} \sqrt{1+\frac{R_{t}}{S L_{t}}}, \quad \text { and } \quad \gamma l=S \sqrt{L_{t} C_{t}} \sqrt{1+\frac{R_{t}}{S L_{t}}} .
$$

For a CMOS gate driving another CMOS gate at the end of the line, $z_{s}=R_{t r}$ and $z_{L}=1 / S C_{L}$. A time scaling is applied by substituting $t^{\prime} /$ $\omega_{n}$ for each $t$ where

$$
\omega_{n}=\frac{1}{\sqrt{L_{t}\left(C_{t}+C_{L}\right)}} .
$$

From the characteristics of the Laplace transform, the complex frequency $S$ is substituted by $\omega_{n} S$ '. With this time scaling, the variables $\gamma, z_{0}$, and $z_{L}$ are transformed to $\gamma, z^{\prime}{ }_{0}$, and $z_{L}{ }_{L}$, respectively, which can be evaluated by substituting $\omega_{n} S$ ' for each $S$. If the exponential functions in the transfer function in (2) are replaced by a series expansion, the transfer function becomes 
$\frac{V_{\text {out }}\left(S^{\prime}\right)}{V_{\text {in }}\left(S^{\prime}\right)}=\frac{1}{\left.\left(1+\frac{z_{s}}{z_{L}^{\prime}}\right)\left(1+\frac{\left(\gamma^{\prime} l\right)^{2}}{2 !}+\ldots . .\right)+\left(\frac{z_{s}}{z_{0}^{\prime}}+\frac{z_{0}^{\prime}}{z_{L}^{\prime}}\right)\left(\gamma^{\prime} l\right)+\frac{\left(\gamma^{\prime} l\right)^{3}}{3 !}+\ldots . .\right)}$.

Substituting for $\gamma^{\prime} l, z^{\prime}{ }_{0}$, and $z^{\prime}{ }_{L}$, the transfer function $V_{\text {out }}\left(S^{\prime}\right) / V_{\text {in }}\left(S^{\prime}\right)$ is a function of only three variables: $\zeta, R_{T}$, and $C_{T}$ which are

$$
\begin{aligned}
& R_{T}=\frac{R_{t r}}{R_{t}}, \quad C_{T}=\frac{C_{L}}{C_{t}}, \quad \text { and } \\
& \zeta=\frac{R_{t}}{2} \sqrt{\frac{C_{t}}{L_{t}}} \cdot \frac{R_{T}+C_{T}+R_{T} C_{T}+0.5}{\sqrt{\left(1+C_{T}\right)}} .
\end{aligned}
$$

The first few terms of the series expansion in $S$ ' are

$$
\frac{V_{\text {out }}\left(S^{\prime}\right)}{V_{\text {in }}\left(S^{\prime}\right)}=\frac{1}{1+2 \zeta S^{\prime}+\left(\frac{0.5+C_{T}}{1+C_{T}}+16 \zeta^{2}\left(1-\frac{R_{T}^{2}+C_{T}^{2}+\left(R_{T} C_{T}\right)^{2}}{\left(R_{T}+C_{T}+R_{T} C_{T}+0.5\right)^{2}}\right)\right) S^{\prime 2}+\cdots} .
$$

Thus, for a unit step input function, the output voltage waveform $V_{\text {out }}\left(t^{\prime}\right)=\mathcal{L}^{-1}\left\{(1 / S)^{*} V_{\text {out }}\left(S^{\prime}\right) / V_{\text {in }}\left(S^{\prime}\right)\right\}$ is also a function of the three variables $\zeta, R_{T}$, and $C_{T}$. The scaled $50 \%$ propagation delay $t^{\prime}{ }_{p d}$ can be calculated by solving $V_{\text {out }}\left(t^{\prime}{ }_{p d}, \zeta, R_{T}, C_{T}\right)=0.5$ which means that $t^{\prime}{ }_{p d}$ is only a function of $\zeta, R_{T}$, and $C_{T}$. Thus, the propagation delay of an $R L C$ line with a source resistance $R_{t r}$ and a load capacitance $C_{L}$ has the form,

$$
t_{p d}=\frac{t_{p d}^{\prime}\left(\zeta, R_{T}, C_{T}\right)}{\omega_{n}} .
$$

Note that this solution is characteristic of an $R L C$ line and that no approximations have been made in deriving the result.

The scaled propagation delay $t_{p d}^{\prime}$ is dimensionless since $\omega_{n}$ has the units of $1 /$ time. $t_{p d}^{\prime}$ is a function of only three variables which is the canonical number of variables to describe $t^{\prime}{ }_{p d}$. There are several ways to select these three variables. The three variables chosen here are $R_{T}, C_{T}$, and $\zeta$ since these variables are physically intuitive. The variables $R_{T}$ and $C_{T}$ characterize the relative significance of the gate parasitic impedances with respect to the interconnect parasitic impedances. Increasing $R_{T}$ and $C_{T}$ demonstrates that the gate parasitic impedances further affect the propagation delay. The third variable $\zeta$ is the coefficient of $S^{l}$ in the denominator of the transfer function. $\zeta$ is chosen as the third variable since the $50 \%$ delay is primarily dependent upon the coefficients of $S^{l}$ in the denominator and the numerator [13]. This characteristic is used to reduce the number of variables that affect the propagation delay from three to one ( $\zeta$ ). Note that the three variables $R_{T}, C_{T}$, and $\zeta$ are not independent since $\zeta$ is a function of $R_{T}$ and $C_{T}$.

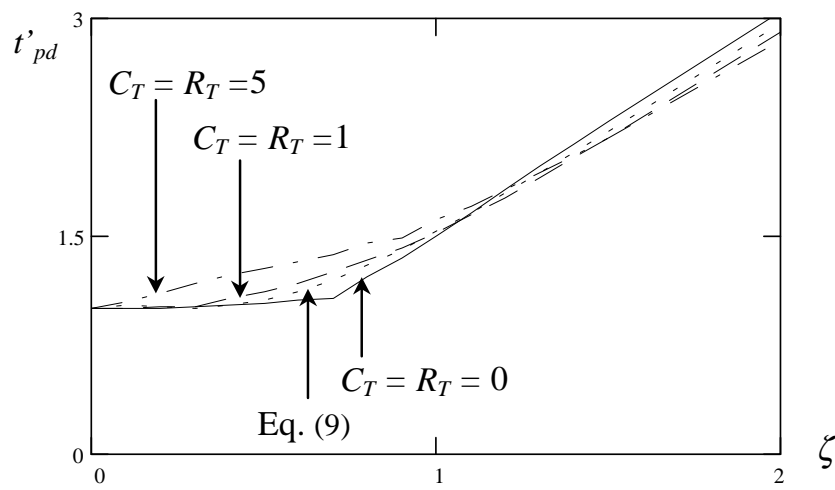

Fig. 2. Comparison of the accuracy of (9) to AS/X [14] simulations of the time scaled $50 \%$ propagation delay $t_{p d}{ }_{p d}$ of an $R L C$ transmission line with a source resistance $R_{t r}$ and a load capacitance $C_{L}$. The propagation delay is plotted versus $\zeta$ for different values of $R_{T}$ and $C_{T}$.

AS/X [14] simulations of the time scaled $50 \%$ propagation delay of a gate driving an $R L C$ transmission line $t_{p d}^{\prime}$ as a function of $\zeta, R_{T}$, and $C_{T}$ are shown in Fig. 2. Note in Fig. 2 that the propagation delay is primarily a function of $\zeta$. The dependence on $R_{T}$ and $C_{T}$ is fairly weak. This characteristic does not imply that the transistor driving the interconnect and the load capacitance has a minor effect on the propagation delay since $\zeta$ includes the effects of $R_{T}$ and $C_{T}$. Note also that this effect is particularly weak in the range where $R_{T}$ and $C_{T}$ are between zero and one. This range is most important for global interconnect and long wires in current deep submicrometer technologies. Thus, the propagation delay is primarily a function of $\zeta$, which collects the five impedances that affect the propagation delay, $R_{t}, L_{t}, C_{t}, R_{t r}$, and $C_{L}$, into a single parameter. A curve fitting method is used to minimize the error when $R_{T}$ and $C_{T}$ are between zero and one, as illustrated in Fig. 2.

Using this approach, the propagation delay in the linear region can be modeled by the following function,

$$
t_{p d}=\left(e^{-2.9 \zeta^{1.35}}+1.48 \zeta\right) / \omega_{n} .
$$

AS/X [14] simulations of the propagation delay of an $R L C$ transmission line as compared to $t_{p d}$ in (9) are shown in Table 1. Note that the solution exhibits high accuracy (the error is less than $5 \%)$ for a wide range of interconnect $\left(R_{t}, L_{t}\right.$, and $\left.C_{t}\right)$ and gate impedances $\left(R_{t r}\right.$ and $\left.C_{L}\right)$. Note also that the simulation data listed in Table 1 include those cases where the response is underdamped and overshoots occur (high inductive effects), and those cases where the response is overdamped (low inductive effects). All of these

\begin{tabular}{|c|c|c|c|c|c|c|c|c|c|c|}
\hline \multirow{2}{*}{$R_{T}$} & \multirow{2}{*}{$\begin{array}{l}L_{t} \\
\mathrm{H}\end{array}$} & \multicolumn{3}{|c|}{$C_{T}=0.1$} & \multicolumn{3}{|c|}{$C_{T}=0.5$} & \multicolumn{3}{|c|}{$C_{T}=1.0$} \\
\hline & & (9) & ASX & Error & (9) & ASX & Error & (9) & ASX & Error \\
\hline \multirow{4}{*}{0.1} & $10^{-5}$ & 3389 & 3287 & $3.3 \%$ & 3893 & 3782 & $2.9 \%$ & 4469 & 4344 & $2.8 \%$ \\
\hline & $10^{-6}$ & 1062 & 1071 & $0.8 \%$ & 1277 & 1328 & $3.8 \%$ & 1553 & 1627 & $4.5 \%$ \\
\hline & $10^{-7}$ & 532 & 552 & $3.6 \%$ & 848 & 881 & $3.7 \%$ & 1248 & 1269 & $1.6 \%$ \\
\hline & $10^{-8}$ & 508 & 496 & $2.4 \%$ & 850 & 883 & $3.7 \%$ & 1239 & 1261 & $1.7 \%$ \\
\hline \multirow{4}{*}{0.5} & $10^{-5}$ & 3397 & 3304 & $2.8 \%$ & 4086 & 3940 & $3.8 \%$ & 4504 & 4518 & $0.3 \%$ \\
\hline & $10^{-6}$ & 1145 & 1108 & $3.3 \%$ & 1489 & 1509 & $1.3 \%$ & 1946 & 2030 & $4.1 \%$ \\
\hline & $10^{-7}$ & 854 & 861 & $0.8 \%$ & 1297 & 1300 & $0.2 \%$ & 1812 & 1830 & $1.0 \%$ \\
\hline & $10^{-8}$ & 841 & 850 & $1.0 \%$ & 1277 & 1283 & $0.5 \%$ & 1811 & 1825 & $0.8 \%$ \\
\hline \multirow{4}{*}{1.0} & $10^{-5}$ & 3397 & 3291 & $3.0 \%$ & 3897 & 3773 & $3.3 \%$ & 4496 & 4383 & $2.6 \%$ \\
\hline & $10^{-6}$ & 1070 & 1076 & $0.6 \%$ & 1323 & 1345 & $1.6 \%$ & 1712 & 1702 & $0.6 \%$ \\
\hline & $10^{-7}$ & 634 & 609 & $4.1 \%$ & 930 & 910 & $2.2 \%$ & 1297 & 1281 & $1.2 \%$ \\
\hline & $10^{-8}$ & 630 & 622 & $1.2 \%$ & 936 & 913 & $2.5 \%$ & 1294 & 1271 & $1.8 \%$ \\
\hline
\end{tabular}
operating modes are described by one continuous equation, (9).

Table 1. Comparison of $t_{p d}$ in (9) to AS/X simulations characterizing the propagation delay of a CMOS gate driving an $R L C$ transmission line. $C_{t}=1 \mathrm{pF}$ and $R_{t r}=500 \Omega$.

An interesting special case is when the gate parasitic impedances $\left(C_{L}\right.$ and $\left.R_{t r}\right)$ are neglected. This case is particularly important since it describes the propagation delay characteristics of a distributed $R L C$ line without the distortion of the gate impedances. For the limiting case where $L \rightarrow 0$, (9) reduces to $0.37 R C l^{2}$. This expression is the same formula for the propagation delay of a distributed $R C$ line as described in [3] and [11]. Also note the well known square dependence on the length of the wire. For the other limiting case where $R \rightarrow 0$, the propagation delay is given by $l \sqrt{L C}$. Note the linear dependence on the length of the line. Thus, the traditional quadratic dependence of the propagation delay on the length of an $R C$ line approaches a linear dependence as inductance effects increase.

\section{Repeater Insertion in $R L C$ Interconnect}

Traditionally, repeaters are inserted into $R C$ lines to partition an interconnect line into shorter sections, e.g., [9]-[11], thereby 
reducing the total propagation delay. Applying the same idea to the general case of an $R L C$ line, repeaters are used to divide the interconnect line into $k$ sections as shown in Fig. 3. The buffers are each uniformly the same size and $h$ times larger than a minimum size buffer. The buffer output impedance $R_{t r}$ is $R_{0} / h$ and the input capacitance of the buffer $C_{L}$ is $h C_{0}$.

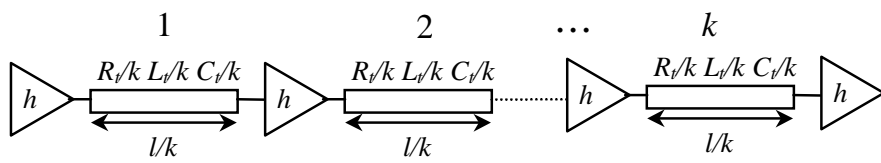

Fig. 3. Repeaters inserted in an $R L C$ line to minimize the propagation delay.

The total propagation delay of a repeater system is the sum of the individual propagation delays of the $k$ sections and is a function of $h$ and $k$ for a given interconnect line. The values of $h$ and $k$ at which the total delay $t_{\text {pdtotal }}$ is a minimum are determined by simultaneously solving the following two differential equations,

$$
\frac{\partial t_{\text {pdtotal }}(h, k)}{\partial h}=0 \quad \text { and } \quad \frac{\partial t_{p d t o t a l}(h, k)}{\partial k}=0 .
$$

For the special case of an $R C$ line $\left(L_{\mathrm{t}} \rightarrow 0\right)$, the solution for these equations is

$$
h_{\text {opt }}(R C)=\sqrt{\frac{R_{0} C_{t}}{R_{t} C_{0}}} \quad \text { and } \quad k_{\text {opt }}(R C)=\sqrt{\frac{R_{t} C_{t}}{2 R_{0} C_{0}}} .
$$

These equations are the same as described by Bakoglu in [11].

Solving (10) for the general case of an $R L C$ line is analytically intractable. However, as described in the appendix, $h_{\text {opt }}$ and $k_{\text {opt }}$ for an $R L C$ line have the form,

$$
h_{\text {opt }}=\sqrt{\frac{R_{0} C_{t}}{R_{t} C_{0}}} \bullet h^{\prime}\left(T_{L / R}\right) \quad \text { and } \quad k_{\text {opt }}=\sqrt{\frac{R_{t} C_{t}}{2 R_{0} C_{0}}} \bullet k^{\prime}\left(T_{L / R}\right) \text {, }
$$

where $h^{\prime}\left(T_{L / R}\right)$ and $k^{\prime}\left(T_{L / R}\right)$ are error factors that account for the effect of the inductance and $T_{L / R}$ is

$$
T_{L / R}=\sqrt{\frac{L_{t} / R_{t}}{R_{0} C_{0}}} .
$$

The closed form solution for the propagation delay in (9) is used to characterize the delay of the repeater system shown in Fig. 3 as described in the appendix. The resulting expression is partially differentiated with respect to $h$ and $k$ and the two derivatives are equated to zero. The resulting two equations are solved numerically for the optimum values of $h$ and $k$. Numerical solutions for $h_{o p t}$ and $k_{\text {opt }}$ in (10) for different values of $T_{L / R}$ are plotted in Fig. 4.

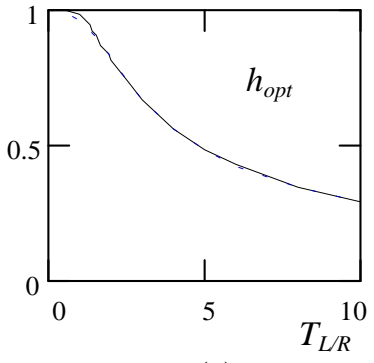

(a)

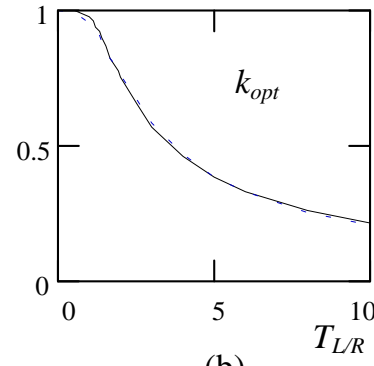

(b)
Fig. 4. Numerical solutions of (10) as compared to eqs. (14) and (15). a) $h_{\text {opt }}$ as compared to (14). b) $k_{\text {opt }}$ as compared to (15). Numerical solutions are shown by the solid line while (14) and (15) are shown by the dashed line.

Curve fitting is employed to determine a function that accurately characterizes $h_{\text {opt }}$ and $k_{\text {opt }}$. These functions are

$$
h_{\text {opt }}=\sqrt{\frac{R_{0} C_{t}}{R_{t} C_{0}}} \frac{1}{\left[1+0.16\left(T_{L / R}\right)^{3}\right]^{0.24}},
$$

and

$$
k_{\text {opt }}=\sqrt{\frac{R_{t} C_{t}}{2 R_{0} C_{0}}} \frac{1}{\left[1+0.18\left(T_{L / R}\right)^{3}\right]^{0.3}} .
$$

These closed form expressions are highly accurate with an error of the total propagation delay of the repeater system of less than $0.05 \%$ as compared to numerical analysis. These formulae can therefore be considered exact for all practical purposes.

Upon examination of (14) and (15), $h_{\text {opt }}$ and $k_{\text {opt }}$ are equal to $h_{\text {opt }}(R C)$ and $k_{\text {opt }}(R C)$ in (11) for the special case of an $R C$ impedance when $L_{\mathrm{t}} \rightarrow 0$ (or $T_{L R} \rightarrow 0$ ). Note that the error between the two cases increases as $T_{L / R}$ increases. This behavior is understandable since inductance effects are more significant as $T_{L / R}$ increases (which increases the error of neglecting $L_{t}$ ). Also note that as $T_{L / R}$ increases (or the inductance effects increase), the number of sections $k_{\text {opt }}$ decreases. The improvement achieved by partitioning the line into shorter sections in the $R C$ case is primarily due to the quadratic dependence of the propagation delay on interconnect length. In the other extreme case of an $L C$ line, the propagation delay is linear with interconnect length and therefore no speed improvement is achieved by partitioning the line into shorter subsections. Actually, adding repeaters in this case would only increase the total propagation delay because of the additional gate delay of the repeaters. Thus, as inductance effects increase, the optimum number of repeaters to insert to minimize the total interconnect delay decreases.

The per cent increase in $t_{\text {pdtotal }}$ caused by neglecting inductance and treating an $R L C$ line as an $R C$ line as compared to including inductance based on (14) and (15) for $h_{\text {opt }}$ and $k_{\text {opt }}$, respectively, is

$$
\% \text { Increase }=\frac{100 *\left\lfloor\left(t_{\text {pdtotal }}\right)_{R C}-\left(t_{\text {pdtotal }}\right)_{R L C}\right\rfloor}{\left(t_{\text {pdtotal }}\right)_{R L C}} .
$$

$\left(t_{p d t o t a l}\right)_{R C}$ is calculated by substituting the solution for $h_{o p t}(R C)$ and $k_{\text {opt }}(R C)$ in (11) into $t_{\text {pdtotal }} .\left(t_{\text {pdtotal }}\right)_{R L C}$ is calculated by substituting the solution for $h_{\text {opt }}$ and $k_{\text {opt }}$ in (14) and (15), respectively, into $t_{\text {pdtotal }}$. The resulting solution is a function of $T_{L / R}$ only and can be accurately approximated by

$$
\% \text { Increase }=\frac{30}{\sqrt{\left(1+\frac{0.5}{T_{L / R}}+23 e^{-0.8 T_{L / R}}+10^{4} e^{-4 T_{L / R}}\right)}} .
$$

Note that $\left(t_{\text {pdtotal }}\right)_{R C}$ is larger than $\left(t_{\text {pdtotal }}\right)_{R L C}$ as $T_{L / R}$ increases. For $T_{L / R}=3, t_{\text {pdtotal }}$ increases by $10 \%$, for $T_{L / R}=5, t_{\text {pdtotal }}$ increases by $20 \%$, and for $T_{L / R}=10, t_{\text {pdtotal }}$ increases by $30 \%$.

The total area of the buffers in the repeater system is given by $A_{R L C}=h_{\text {opt }} * k_{\text {opt }} * A_{\text {min }}$ and $A_{R C}=h_{\text {opt }}(R C) * k_{\text {opt }}(R C) * A_{\text {min }}$ for the $R L C$ and the $R C$ case, respectively. $A_{\min }$ is the area of a minimum size buffer. The per cent area increase $\% A I$ is characterized by $100 *\left(A_{R C^{-}}\right.$ $\left.A_{R L C}\right) / A_{R L C}$ and is

$$
\% A I=100 \cdot\left\{\left[1+0.18\left(T_{L / R}\right)^{3}\right]^{0.3} \cdot\left[1+0.16\left(T_{L / R}\right)^{3}\right]^{0.24}-1\right\} .
$$

The per cent area increase for $T_{L / R}=3$ is $154 \%$ and for $T_{L / R}=5$ is $435 \%$. From the impedance values described in [7], it can be shown that $T_{L / R}=5$ is common for a current $0.25 \mu \mathrm{m}$ technology. Thus, neglecting inductance not only increases the total delay of the repeater system but significantly increases the buffer area as well. This trend is expected since treating the interconnect as an $R C$ line and neglecting inductance requires more repeaters. These additional repeaters add to the total delay and buffer area without reducing the line delay because significant inductance makes the dependence of the delay on the length of the interconnect become sub-quadratic. Note that $T_{L / R}$ increases as $R_{0} C_{0}$ decreases. This relation means that as the gate delay decreases, inductance becomes more important. 
Thus, the effects of inductance in next generation design methodologies will become fundamentally important as technologies scale.

\section{Conclusions}

Closed form solutions for the propagation delay of a CMOS gate driving a distributed $R L C$ load are presented that are within 5\% of AS/X simulations. It is shown that the traditional quadratic dependence of the propagation delay on the length of an $R C$ line tends to a linear dependence as inductance effects increase. This behavior is expected to have a profound effect on future high speed CMOS technologies.

Closed form solutions are presented for inserting repeaters into $R L C$ lines that are highly accurate with respect to numerical solutions. Inserting repeaters based on an $R C$ model into $R L C$ lines as compared to applying a distributed $R L C$ impedance model of the interconnect increases the propagation delay by up to $30 \%$, and the repeater area by up to $435 \%$ for common VLSI interconnect. The power consumption of the repeater system is also expected to be much less in the case of an $R L C$ model as compared to an $R C$ model due to the increased repeater area for the $R C$ case. Thus, incorporating inductance into the interconnect impedance model is of crucial importance for accurately estimating the propagation delay of on-chip interconnect as well as for minimizing the propagation delay. This importance is expected to increase as the gate parasitic impedances decrease and as technologies increase in speed.

\section{Appendix}

\section{Optimum Repeater Insertion in $R L C$ Lines}

As shown in section II, the propagation delay of a gate driving a single section of interconnect with an impedance of $R_{t}, C_{t}$, and $L_{t}$ has the form given by (8). If repeaters are inserted to partition the line into $k$ sections and each repeater is $h$ times greater than a minimum size inverter, the total propagation delay of the system is the summation of the propagation delays of each of the individual sections. Since the sections are each equal, the total delay can be expressed as $t_{p d t o t a l}=k t_{p d s e c}$, where $t_{p d s e c}$ is the propagation delay of a single section. Each section has an interconnect impedance equal to $R_{t} / k, C_{t} / k$, and $L_{t} / k$. Since each repeater is $h$ times larger than a minimum size buffer, each repeater has an output resistance $R_{t r}=R_{0} /$ $h$ and a load capacitance of $C_{L}=C_{0} h$. Thus, the total propagation delay of the repeater system is

$$
t_{p d t o t a l}=k \cdot \frac{i_{p d}^{\prime}\left(\zeta_{\mathrm{sec}}, R_{T \mathrm{sec}}, C_{T \mathrm{sec}}\right)}{\omega_{n \mathrm{sec}}},
$$

where $R_{T s e c}$ and $C_{T s e c}$ are

$$
R_{T \mathrm{sec}}=\frac{k}{h} \frac{R_{0}}{R_{t}} \quad \text { and } \quad C_{T \mathrm{sec}}=k h \frac{C_{0}}{C_{t}} .
$$

$\zeta_{\text {sec }}$ and $\omega_{n s e c}$ are

$$
\begin{gathered}
\zeta_{\mathrm{sec}}=\frac{R_{t}}{2 k} \sqrt{\frac{C_{t}}{L_{t}}} \cdot \frac{R_{T \mathrm{sec}}+C_{T \mathrm{sec}}+R_{T \mathrm{sec}} C_{T \mathrm{sec}}+0.5}{\sqrt{\left(1+C_{T \mathrm{sec}}\right)}}, \\
\omega_{n \mathrm{sec}}=\frac{k}{\sqrt{L_{t} C_{t}} \sqrt{1+C_{T \mathrm{sec}}}} .
\end{gathered}
$$

Guided by the solution of $h$ and $k$ for the special case of an $R C$ line, the solution for an $R L C$ line is in the form of

$$
h=\sqrt{\frac{R_{0} C_{t}}{R_{t} C_{0}}} \bullet h^{\prime} \quad \text { and } \quad k=\sqrt{\frac{R_{t} C_{t}}{2 R_{0} C_{0}}} \bullet k^{\prime},
$$

where $h^{\prime}$ and $k^{\prime}$ are error factors that incorporate the existence of inductance and approach one as the inductance approaches zero. Substituting these values for $h$ and $k$ into (20), (21), and (22), the variables $R_{T s e c}, C_{T s e c}, \zeta_{s e c}$, and $\omega_{n s e c}$ become

$$
R_{T \text { sec }}=\frac{k^{\prime}}{h^{\prime} \sqrt{2}}, \quad C_{T \text { sec }}=\frac{h^{\prime} k^{\prime}}{\sqrt{2}},
$$

$$
\zeta_{\mathrm{sec}}=\frac{1}{\sqrt{2} k^{\prime} T_{L / R}} \cdot \frac{R_{T \mathrm{sec}}+C_{T \mathrm{sec}}+R_{T \mathrm{sec}} C_{T \mathrm{sec}}+0.5}{\sqrt{\left(1+C_{T \mathrm{sec}}\right)}},
$$

and

$$
\omega_{n \text { sec }}=\frac{k}{\sqrt{L_{t} C_{t}} \sqrt{\left(1+C_{T \text { sec }}\right)}},
$$

where $T_{L / R}$ is given by

$$
T_{L / R}=\sqrt{\frac{L_{t} / R_{t}}{R_{0} C_{0}}} .
$$

Thus, the total propagation delay has the form,

$$
t_{\text {pdtotal }}=\sqrt{L_{t} C_{t}} \cdot f\left(h^{\prime}, k^{\prime}, T_{L / R}\right) \cdot
$$

Determining the values of $k^{\prime}$ and $h^{\prime}$ that minimize the total propagation delay requires the simultaneous solution of the following two differential equations,

$$
\frac{\partial f\left(h^{\prime}, k^{\prime}, T_{L / R}\right)}{\partial h^{\prime}}=0 \quad \text { and } \quad \frac{\partial f\left(h^{\prime}, k^{\prime}, T_{L / R}\right)}{\partial k^{\prime}}=0 .
$$

The solution of these equations demonstrates that $h^{\prime}$ and $k^{\prime}$ are only functions of $T_{L / R}$. Thus, the optimum number of sections $k_{\text {opt }}$ and the optimum repeater size $h_{\text {opt }}$ for an $R L C$ interconnect is

$$
h_{\text {opt }}=\sqrt{\frac{R_{0} C_{t}}{R_{t} C_{0}}} \bullet h^{\prime}\left(T_{L / R}\right) \quad \text { and } \quad k_{\text {opt }}=\sqrt{\frac{R_{t} C_{t}}{2 R_{0} C_{0}}} \bullet k^{\prime}\left(T_{L / R}\right) \text {. }
$$

Note that this solution is characteristic of an $R L C$ line and that no approximations have been made in deriving this result.

\section{References}

[1] D. A. Priore, "Inductance on Silicon for Sub-Micron CMOS VLSI," Proceedings of the IEEE Symposium on VLSI Circuits, pp. 17-18, May 1993.

[2] M. P. May, A. Taflove, and J. Baron, "FD-TD Modeling of Digital Signal Propagation in 3-D Circuits with Passive and Active Loads," IEEE Transactions on Microwave Theory and Techniques, Vol. MTT-42, No. 8, pp. 1514 - 1523, August 1994.

[3] T. Sakurai, "Approximation of Wiring Delay in MOSFET LSI," IEEE Journal of Solid-State Circuits, Vol. SC-18, No. 4, pp. 418 - 426, August 1983.

[4] G. Y. Yacoub, H. Pham, and E. G. Friedman, "A System for Critical Path Analysis Based on Back Annotation and Distributed Interconnect Impedance Models," Microelectronic Journal, Vol. 18, No. 3, pp. 21 - 30, June 1988.

[5] M. Shoji, High-Speed Digital Circuits, Addison Wesley, Massachusetts, 1996

[6] J. Torres, "Advanced Copper Interconnections for Silicon CMOS Technologies," Applied Surface Science, Vol. 91, No. 1, pp. 112 123, October 1995.

[7] A. Deutsch et al., "When are Transmission-Line Effects Important for On-Chip Interconnections?," IEEE Transactions on Microwave Theory and Techniques, Vol. MTT-45, No. 10, pp. 1836-1846, October 1997.

[8] Y. I. Ismail, E. G. Friedman, and J. L. Neves, "Figures of Merit to Characterize the Importance of On-Chip Inductance," Proceedings of the IEEE/ACM Design Automation Conference, pp. 560 - 565, June 1998.

[9] H. B. Bakoglu and J. D. Meindl, "Optimal Interconnection Circuits for VLSI," IEEE Transactions on Electron Devices, Vol. ED-32, No. 5, pp. 903 - 909, May 1985.

[10] V. Adler and E. G. Friedman, "Repeater Design to Reduce Delay and Power in Resistive Interconnect," IEEE Transactions on Circuits and Systems II: Analog and Digital Signal Processing, Vol. CAS-45, No. 5, pp. 607 - 616, May 1998.

[11] H. B. Bakoglu, Circuits, Interconnections, and Packaging for VLSI, Addison-Wesley Publishing Company, 1990.

[12] L. N. Dworsky, Modern Transmission Line Theory and Applications, John Wiley \& Sons, Inc., New York, 1979.

[13] W. C. Elmore, "The Transient Response of Damped Linear Networks," Journal of Applied Physics, Vol. 19, pp. 55 - 63, January 1948.

[14] AS/X User's Guide, IBM Corporation, New York, 1996. 